\title{
Abstract Elementary Classes: Some Answers, More Questions
}

\author{
John T. Baldwin* \\ Department of Mathematics, Statistics and Computer Science \\ University of Illinois at Chicago
}

July 7, 2006

\begin{abstract}
We survey some of the recent work in the study of Abstract Elementary Classes focusing on the categoricity spectrum and the introduction of certain conditions (amalgamation, tameness, arbitrarily large models) which allow one to develop a workable theory. We repeat or raise for the first time a number of questions; many now seem to be accessible.
\end{abstract}

Much late 19th and early 20th century work in logic was in a 2nd order framework; infinitary logics in the modern sense were foreshadowed by Schroeder and Pierce before being formalized in modern terms in Poland during the late 20's. First order logic was only singled out as the 'natural' language to formalize mathematics as such authors as Tarski, Robinson, and Malcev developed the fundamental tools and applied model theory in the study of algebra. Serious work extending the model theory of the 50's to various infinitary logics blossomed during the 1960's and 70's with substantial work on logics such as $L_{\omega_{1}, \omega}$ and $L_{\omega_{1}, \omega}(Q)$. At the same time Shelah's work on stable theories completed the switch in focus in first order model theory from study of the logic to the study of complete first order theories As Shelah in [She75, She83a] sought to bring this same classification theory standpoint to infinitary logic, he introduced a total switch to a semantic standpoint. Instead of studying theories in a logic, one studies the class of models defined by a theory. He abstracted (pardon the pun) the essential features of the class of models of a first order theory partially ordered by the elementary submodel relation. An abstract elementary class AEC $(\boldsymbol{K}, \prec \boldsymbol{K})$ is a class of models closed under isomorphism and partially ordered under $\prec \boldsymbol{K}$, where $\prec_{\boldsymbol{K}}$ is required to refine the substructure relation, that is closed under unions and satisfies two additional conditions: if each element $M_{i}$ of a chain satisfies $M_{i} \prec{ }_{K} M$ then $M_{0} \prec_{\boldsymbol{K}} \bigcup_{i} M_{i} \prec \boldsymbol{K} M$ and $M_{0} \prec \boldsymbol{K} M_{2}, M_{1} \prec \boldsymbol{K} M_{2}$ and $M_{0} \subseteq M_{1}$ implies $M_{0} \prec \boldsymbol{K} M_{1}$ (coherence axiom). Further there is a Löwnenheim-Skolem number $\kappa$ associated with $\boldsymbol{K}$ so that if $A \subseteq M \in \boldsymbol{K}$, there is an $M_{1}$ with $A \subset M_{1} \prec \boldsymbol{K} M$ and $\left|M_{1}\right| \leq|A|+\kappa$.

\footnotetext{
*Partially supported by DMS -0500841
} 
In this paper we will review some of the reasons for considering AEC's, outline several major lines of study in the subject, and offer a series of problems whose solution would advance the various lines. The fundamental ideas discussed here are due to Shelah. However, we explore in some detail areas that have been developed in the very recent past by such authors as Grossberg, Hyttinen, Kolesnikov, Lessmann, VanDieren, and Villaveces; generally speaking these studies proceed by putting further model theoretic conditions on an AEC and we will expound some of these conditions. In the closing pages we give a short introduction to the mainline of Shelah's research [She01, She0x, She00b, She00a].

Our survey focuses primarily on problems closely related to categoricity. We have attempted to attribute both results and questions correctly. But many of the questions are just writing out what people in the area are thinking about. For expositional purposes, we frequently cite [Bal]; the default is that results in that monograph are not new although the proofs may be. I thank Tapani Hyttinen, the anonymous referee, and especially Rami Grossberg for useful comments on this article.

Increased interest in nonelementary classes arose recently for several reasons. First, the increased emphasis, signaled in [She99, SV99] and emphasized in [GV06a], on hypotheses such as amalgamation or tameness as fruitful conditions to create a workable theory of AEC, has led to a number of new results. The need for studying AEC became more clear for two reasons. On the one hand the pursuit of specific problems in the first order setting has led to constructions which can no longer be formalized by first order means. On the other, the paradigm: study an interesting structure by studying its first order theory has broken down in some significant cases because the first order theory is not sufficiently nice.

The work of Kim and Pillay [KP97] showed that the essential distinction between stable and simple theories [She80] lay in the fact that for a stable theory, Lascar strong type equals strong type. Strong types are first order objects; Lascar strong types are not. Analysis of this problem led to the introduction of hyperimaginaries and other properly infinitary objects and ultimately to compact abstract theories CATS [BY03b]. In a slightly different direction, the 'Hrushovski construction' [Hru93, Hru92] leads in nice cases (when the generic is $\omega$-saturated) to the construction of first order theories with special properties. However, in certain notable cases, the best that has so far been found is a Robinson theory (in the search for a bad field [Bal04, BH00]) or even only a positive Robinson theory (in the search for a simple theory where strong type is not equal to Lascar strong type [PW0x]). Despite the terminology, a (positive) Robinson theory, refers to the class of models of a first order theory which omit certain types; it can be described only in infinitary logic.

The first order theory of the field of complex numbers with exponentiation is intractable; the ring of integers and their order is first order definable. But Zilber suggested in a sequence of papers [Zil05, Zil04, Zil00, Zil02] the notion of considering the $L_{\omega_{1}, \omega}(Q)$-theory of $(\mathbb{C},+, \cdot, \exp )$. The intuition is that the essential wildness will be contained by forcing the kernel of the exponential map to always be exactly the standard integers. In his proof of categoricity 
for quasiminimal excellent classes Zilber discovered a special case of Shelah's notion of excellence that is easy to describe. He works in a context where there is a well-behaved notion of closure, cl which defines a combinatorial geometry. The aim is to show that if $X$ is isomorphic to $Y$, then $\operatorname{cl}(X)$ is isomorphic to $\mathrm{cl}(Y)$. In general, this condition is non-trivial; it follows from excellence. In this context, excellence means that for every $n$, if $A=\left\{a_{1}, \ldots a_{n}\right\}$ is an independent set then for any $a \in \operatorname{cl}(A)$ the type of $a$ over $Z=\bigcup_{i<n} \operatorname{cl}\left(A-\left\{a_{i}\right\}\right)$ is determined by the type of $a$ over a finite subset of $Z$. Shelah works in a more general situation, where combinatorial geometry is replaced by a 'forking'like notion. Consequently his notion is harder to describe and we omit the description here. Crucially, in both cases a condition (excellence) on countable models has important consequences (e.g. amalgamation) in all cardinalities.

Various other attempts to formalize analytic structures (notably Banach spaces [Hen72, HI02]) provide examples of 'homogeneous model theory' ([She70, BL03] and many more); Banach spaces are also an example of CATS [BY03a]. Strictly speaking, the class of Banach spaces is not closed under unions of chains so doesn't form an AEC. But, Banach space model theory can be thought of as the study not of Banach spaces, but of structures whose completion is a Banach Space and this provides an interpretation of classes of Banach spaces as AEC's. Further mathematical examples include locally finite groups [GS83] and some aspects of compact complex manifolds (Although here, the first order theory is an attractive topic for model theorists (e.g. [Moo, Rad04]).).

Many, but not all, of these 'infinitary' formalizations can be captured in the framework of AEC's. (In particular, CATS are inherently different.) The work that I'll describe here has a complementary motivation. Stability theory provided a very strong tool to classify first order theories and then for extremely well-behaved theories (those below the 'main gap') to assign invariants to models of the theories. This insight of Shelah spread beyond stable theories with the realization that very different tools but some of the same heuristics allowed the study of o-minimal theories. By these techniques, o-minimality and stability, model theorists have learned much about the theories of both the real and the complex numbers and many other algebraic structures. But Shelah asks an in some ways more basic question. What are the properties of first order logic that make stability theory work? To what extent can we extend our results to wider classes, in particular to AEC?

Most known mathematical results are either extremely cardinal dependent: about finite or countable structures or at most structures of cardinality the continuum; or completely cardinal independent: about every structure satisfying certain properties. Already first order model theory has discovered problems that have an intimate relation between the cardinality of structures and algebraic properties of the structures:

1. Stability spectrum and counting models

2. A general theory of independence

3. Decomposition theorems for general models 
There are structural algebraic, not merely combinatorial features, which are non-trivially cardinal dependent. (For example, the general theory of independence is intimately related with the class of cardinals in which the theory is stable and even for stable first order theories, stability in $\kappa$ depends on the cofinality of $\kappa$.)

As usual a class of models $\boldsymbol{K}$ with a distinguished notion of submodel has joint embedding property (jep) if any two members of $\boldsymbol{K}$ have a common extension and $\boldsymbol{K}$ has the amalgamation property (ap) if any two extensions of a fixed model $M$ have a common extension (over $M$ ).

If we were to take the fundamental analogy to be that an abstract elementary class represents a complete first order theory then we would add to the definition that the class $(\boldsymbol{K}, \prec \boldsymbol{K})$ has the amalgamation and the joint embedding property. But completeness is a bit much to ask even in $L_{\omega_{1}, \omega}$. Here completeness (all models Karp equivalent) is not necessarily compatible with Löwenheim number $\omega$. Some uncountable models do not have countable Karp equivalent submodels. The standard first order proof of the theorem, 'categoricity in power implies completeness' is a triviality but it assumes both the upwards and downwards Löwenheim-Skolem theorem for a set of sentences. Even for a sentence of $L_{\omega_{1}, \omega}$ in a countable language the reduction for an arbitrary categorical sentence $\psi$ to one which is complete and has essentially the same spectrum is not at all trivial [She75, She83a]. It is substantially easier if $\psi$ is assumed to have arbitrarily large models ([Bal] VII.2) than without that hypothesis ([Bal] VII.3). The difficult case is carried out in full in [She75, She83a]; the easier case is hinted at in [She75, She83a] but spelled out in the expository [Bal04, Bal]. In either case a notion of stability (counting the number of types) is used to obtain even the completeness result.

Moreover, unlike the first order case, completeness does not immediately yield the amalgamation property. The only known proof [She83a, She83b] that a categorical sentence in $L_{\omega_{1}, \omega}$ has the amalgamation property invokes the weak continuum hypothesis and introduces the much more intricate notion of excellence. Moreover few models in every cardinal up to $\aleph_{\omega}$ is assumed; indeed, categoricity in every cardinal up to $\aleph_{\omega}$ is essential to get eventual categoricity [HS90, Bal]. Similarly, although Zilber's quasiminimal excellent classes do have the amalgamation property the existing proof deduces the result from the proof of excellence, which has non-trivial algebraic content (e.g [Zil00]).

We will discuss first AEC with arbitrarily large models and then move to a harder case where that assumption is not made.

Question 1 Must the class of models of a sentence in $L_{\omega_{1}, \omega}$ (or more generally an $A E C$ ) that has arbitrarily large models and is categorical in a sufficiently large cardinal have the amalgamation property (at least for sufficiently large models). This is an interesting question even assuming the weak GCH; the necessity of such an assumption presents a different set of problems.

Grossberg (e.g [Gro02]) has posed this question for AEC and for $L_{\omega_{1}, \omega}$. For sentences of $L_{\omega_{1}, \omega}$, Shelah's result reported above gives a partial answer modulo 
weak gch. He deduces excellence and thus amalgamation from categoricity up to $\aleph_{\omega}$. But although Grossberg's question is on the 'assume arbitrarily large models side', it is more demanding than Shelah's result in asking that categoricity in one cardinality suffice. Trying to obtain a proof (even for $L_{\omega_{1}, \omega}$ ) from the arbitrarily large model assumption without passing through excellence is a 'warm-up' strategy for the AEC version.

Shelah's presentation theorem is a crucial tool for the study of AEC. It asserts that every AEC $\boldsymbol{K}$ may be seen as the class of reducts of a collection of models defined by a first order theory (in a language of size $\operatorname{LS}(\boldsymbol{K})$ ) which omit a specified collection of (at most $\left.2^{\mathrm{LS}(\boldsymbol{K})}\right)$ types. Let us state a crucial corollary. Fix a vocabulary $\tau$. For each pair of a first order theory and set of types $\Phi$ (in a vocabulary $\tau^{\prime}$ extending $\tau$ ), and each linear order $I, E M(I, \Phi)$ denotes the reduct to $\tau$ of the $\tau^{\prime}$-structure which satisfies $\Phi$. The presentation theorem implies that for each $\boldsymbol{K}$, there is a $\Phi$ such that $E M\left({ }_{-}, \Phi\right)$ is a functor into $\boldsymbol{K}$ (which takes subordering to $\prec \boldsymbol{K}$ ). A straightforward use of Ehrenfeucht-Mostowski models over indiscernibles yields: If $\boldsymbol{K}$ has a model of cardinality greater than $\beth_{\left(2^{\mathrm{LS}}\left(\boldsymbol{K}_{)}\right)^{+}\right.}$then $\boldsymbol{K}$ has arbitrarily large models. In the vernacular, we say the Hanf number for AEC with vocabulary of size at most $\kappa$ and Löwenheim-Skolem number at most $\kappa$ is at most $H(\kappa)=\beth_{\left(2^{\kappa}\right)^{+}}$. We call this function $H$ as we use it to compute Hanf numbers. It might be more appropriate to call it ER as it actually computes the bound for applying the Erdos-Rado theorem to obtain indiscernibles.

Many of the ideas expounded here were presaged in earlier work such as [MS90, KS96] dealing with languages $L_{\kappa, \omega}$ with strong hypotheses (e.g. compactness, measurability) on the cardinal $\kappa$. The earliest result in this series was:

Theorem 2 [MS90] Let $\kappa$ be strongly compact. If a sentence $\psi \in L_{\kappa, \omega}$ is categorical in $\lambda^{+}>\mu_{0}=\beth_{\left(2^{\kappa}\right)^{+}}$then it is categorical in all cardinals greater than $\mu_{0}$.

In view of the set-theoretic requirements on the syntax of the underlying logic, we don't discuss this line but deal with the more general notion of AEC.

For most of the rest of this paper, we will assume $\boldsymbol{K}$ is an AEC with the amalgamation property. It is then trivial to reduce to the study of AEC with both the amalgamation and joint embedding properties. Under these hypotheses, when $\boldsymbol{K}$ has arbitrarily large models, we are able to work inside a monster model which is behaves much like the first order situation but is weaker in a significant way. We have amalgamation only over submodels, thus the monster model is homogeneous only over submodels. The stronger condition, assuming that there is a 'monster model' that is homogeneous over sets, gives rise to the area known as homogeneous model theory. For the major literature in this area consult such authors as Hyttinen, Lessmann, and Shelah.

Working within a model-homogeneous 'monster model' (i.e. in an AEC with amalgamation), we define the Galois type of $a$ over $M$ to be the orbit of $a$ under 
automorphisms of the monster which fix $M$. We write $\mathcal{S}(M)$ for the collection of Galois types over $M$. Then we can define a model $M$ to be $\kappa$-saturated if every Galois type over a submodel of $M$ with cardinality $<\kappa$ is realized in $M$. A somewhat more general definition (without assuming ap) occurs in [She87, She99].

We begin by discussing classes which have arbitrarily large models. Invoking the presentation theorem, we are able to build Ehrenfeucht-Mostowski models over sequences of order indiscernibles. As Shelah remarks in the introduction to [She01], this yields the non-definability of well-ordering and so gives us an approximation to compactness. Most of these notes concern this case and build on [She99]. We return at the end to the much more difficult situation, where one attempts to find information about AEC simply from the information that it has one (or few models) in some specific cardinalities. We will sketch some of Shelah's extensive work on this subject; our emphasis on classes with arbitrarily large models represents the extent of our understanding, not importance.

Assuming $\boldsymbol{K}$ has arbitrarily large models, the proof that categoricity in $\lambda$ implies stability in all cardinals smaller than $\lambda$ has the same general form as in the first order case. But, one must replace the Ehrenfeucht-Mostowski hull of a cardinal by the hull of a sufficiently homogeneous linear order and make judicious use of the coherence axiom to carry through the proof [She99, Bal]. Thus, the argument is significantly more complicated. This is in interesting contrast with the Laskowski-Pillay study of 'gross-models' [LP]; a model is gross if every infinite definable subset of it has full cardinality. Morley's theorem can be proved in this context using the normal first order notion of type. Thus, the categoricity implies stability is routine. Intriguingly, the Laskowski-Pillay work was inspired by investigations of Moosa on the first order theory of compact complex manifolds.

The fundamental test question for the study of AEC is:

Conjecture 3 (Shelah's categoricity conjecture) There is a cardinal $\mu(\kappa)$ such that for all $A E C$ with Löwenheim number at most $\kappa$, if $\boldsymbol{K}$ is categorical in some cardinal greater than $\mu(\kappa)$ then $\boldsymbol{K}$ is categorical in all $\lambda \geq \mu(\kappa)$.

The best approximation to the categoricity conjecture takes $\mu(\kappa)$ as the 'second Hanf number': $H_{2}=H(H(\operatorname{LS}(\boldsymbol{K})))$. The initial step in the analysis [She99] (see also [Bal]) requires the lifting to this setting of a clever integration of Morley's omitting types theorem and Morley's two cardinal theorem.

Theorem 4 [She99] Suppose $\boldsymbol{K}$ has the amalgamation property and arbitrarily large models. Suppose $\boldsymbol{K}$ is $\lambda^{+}$categorical with $\lambda>\mathrm{H}_{2}$. Then, $\boldsymbol{K}$ is $\mathrm{H}_{2}$ categorical and indeed categorical on the interval $\left[H_{1}, \lambda^{+}\right]$.

The proof requires using the omitting types theorem twice. The second time one names as many constants $\left(H_{1}\right)$ as required for the first use. Categoricity on the interval is then proved by induction, making essential use of Theorem 7 . Theorem 4 leads to a natural question. 
Question 5 Prove or disprove. Suppose $\boldsymbol{K}$ has the amalgamation property and arbitrarily large models. Suppose $\boldsymbol{K}$ is $\lambda^{+}$-categorical with $\lambda>H_{1}$. Then, $\boldsymbol{K}$ is $H_{1}$-categorical.

In order to understand further progress on the categoricity transfer problem, we introduce an important notion (first named in [GV06a]; the cardinal parameters were added in [Bal05]).

Definition 6 The AEC $\boldsymbol{K}$ is $(\chi, \lambda)$-(weakly) tame if for any (saturated) model $M$ of cardinality $\lambda$, if $p, q \in \mathcal{S}(M)$ (the Galois types over $M$ ) are distinct then there is a submodel $N$ of $M$ with $N \leq \chi$ so that $p\lceil N \neq q \uparrow N$.

Of course any first order theory is tame; i.e. $\left(\aleph_{0}, \infty\right)$-tame. And by [She83a, She83b], it is consistent with ZFC that every categorical AEC defined by a sentence of $L_{\omega_{1}, \omega}$ is tame. But aside from the first order case (and homogeneous model theory where again every class is tame), there is no example where $\left(\aleph_{0}, \infty\right)$-tameness has been deduced from categoricity except as a corollary to the Morley theorem for the class. (E.g. Zilber's quasiminimal excellent classes and categorical classes in $L_{\omega_{1}, \omega}$ are each shown to be tame in [Bal]; but the result is not needed for the transfer of categoricity proof given but only an observation.)

Nontameness can arise in natural mathematical settings. An Abelian group is $\aleph_{1}$-free if every countable subgroup is free. An Abelian group $H$ is Whitehead if every extension of $Z$ by $H$ is free. Shelah constructed (in ZFC) an Abelian group of cardinality $\aleph_{1}$ which is $\aleph_{1}$-free but not a Whitehead group. (See [EM90] Chapter VII.4.) Baldwin and Shelah [BS] code this into an example of nontameness. Essentially a point codes an abelian group which is the right end of a short exact sequence; every countable approximation to the group splits but the whole group does not. Thus the AEC is not $\left(\aleph_{0}, \aleph_{1}\right)$-tame. Baldwin and Shelah [BS] also show that nontameness is essentially a distinct phenomena from non-amalgamation by showing any AEC $\boldsymbol{K}$ which exemplifies a nontameness property (satisfying a mild condition) can be transformed to one which does satisfy amalgamation and still fails the tameness. But this transformation destroys categoricity and even stability. In my view, the most significant (nontrivial) sufficient condition for tameness is due to Shelah:

Theorem 7 [She99] Suppose $\boldsymbol{K}$ has the amalgamation property and arbitrarily large models. Suppose $\boldsymbol{K}$ is $\lambda$-categorical with $\lambda>H_{1}$. For every $\kappa$ with $H_{1} \leq$ $\kappa<\lambda, \boldsymbol{K}$ is $(\chi, \kappa)$-weakly tame for some $\chi<H_{1}$.

The combination of Shelah's downward categoricity argument and the tameness argument gives the result for 'tame' instead of 'weakly tame' if $H_{1}$ is allowed to grow to $\mathrm{H}_{2}$. The argument for Theorem 7 in [She99] is flawed. A short and correct argument due to Hyttinen, correcting and elaborating various exegises given separately by Baldwin and Shelah, appears in [Bal]. This result poses several questions. 
Question 8 Suppose $\boldsymbol{K}$ has the amalgamation property and arbitrarily large models. Suppose $\boldsymbol{K}$ is $\lambda$-categorical with $\lambda>H_{1}$.

1. Is there any way to reduce the upper bound on $\chi$ in Theorem 7 (or find a lower bound above $\operatorname{LS}(\boldsymbol{K}))$ ?

2. Is there any way to replace weakly tame by tame?

3. And most important, (compare 1.16 of [GV06a]), can $\kappa=\lambda$ in Theorem 7?

A positive answer to Question 8.3 would yield a full solution of the categoricity problem for AEC with amalgamation and arbitrarily large models.

Is there any way to weaken the categoricity hypothesis in Theorem 7 to stability?

Question 9 Suppose $\boldsymbol{K}$ has the amalgamation property and arbitrarily large models. Prove or disprove: If $\boldsymbol{K}$ is $\kappa$-stable with $\kappa>H_{1}$ then $\boldsymbol{K}$ is (weakly) $\left(H_{1}, \kappa\right)$-tame.

In the light of Theorem 4 and Theorem 7, it is interesting to examine Question 9 at the successor of the categoricity cardinal in the hypothesis of Theorem 4 . How much would it help to know stability in $\lambda^{++}$?

Shelah speaks rather loosely of locality in various places. We have broken this notion into three precise concepts. Following [GV06a], we have chosen tame as the name of one of these. We call the others locality and compactness. There is considerable to be learned about the relations among the parameterized versions of these notions; the following survey just touchs on some of the natural questions that arise. Essentially, they are a few of the many ways one might make specific the general question, 'Are there AEC which are eventually categorical without the many nice properties such as tameness, excellence, locality of the known examples?'

Definition $10 \quad 1 . K$ has $(\kappa, \lambda)$-local Galois types if for every continuous increasing chain $M=\bigcup_{i<\kappa} M_{i}$ of members of $\boldsymbol{K}$ with $|M|=\lambda$ and for any $p, q \in \mathcal{S}(M)$ : if $p\left\lceil M_{i}=q \uparrow M_{i}\right.$ for every $i$ then $p=q$.

2. Galois types are $(\kappa, \lambda)$-compact in $\boldsymbol{K}$ if for every continuous increasing chain $M=\bigcup_{i<\kappa} M_{i}$ of members of $\boldsymbol{K}$ with $|M|=\lambda$ and every increasing chain $\left\{p_{i}: i<\kappa\right\}$ of members $\mathcal{S}\left(M_{i}\right)$ there is a $p \in \mathcal{S}(M)$ with $p\left\lceil M_{i}=p_{i}\right.$ for every $i$.

The proof of Theorem 7 is very much about tameness rather than locality.

Question 11 Is there any way to replace (weakly) tame by local in Theorem 7?

The constructions in $[\mathrm{BS}]$ that create amalgamation destroy categoricity; can this be avoided? More precisely, 
Question 12 Find an AEC (in a countable language) which is categorical in all uncountable powers, has the amalgamation property, and which is not $\left(\aleph_{0}, \infty\right)$ tame $\left(\right.$ or $\left(\aleph_{0}, \infty\right)$-local).

Grossberg and Kolesnikov [GK] recently completed an important analysis of the relationship between excellence and tameness. They work in classes which are posited to have an independence relation analogous to forking in the first order case. They show in particular that if the class satisfies the extension property for independence, the appropriate version of stationarity and the forking has $<\lambda$-character then $\left(\lambda, \lambda^{+}\right)$-tameness and $\left(\lambda, \lambda^{+}\right)$-locality follow. Further, they show that if $\boldsymbol{K}$ is $\chi$-excellent (under an extended definition for this context), then $\boldsymbol{K}$ is $(\chi, \infty)$-tame. There is no simple test, such as failure of the order property in the first order case, to generate a wealth of examples of classes satisfying these hypotheses. It is not even clear that eventual categoricity yields the properties. But as with tameness, these hypotheses provide a platform on which to develop a stability theory for AEC.

A positive answer to either Question 8.1) or Question 11 would seem to require essentially new methods. The distinction between syntactic (given by a set of formulas in some logic) and semantic or Galois types (given by the ability to amalgamate embedding or as orbits in a suitably homogeneous model) leads to a quest for further examples.

Question 13 What are some AEC's which are not basically given syntactically? Which of the many examples of extended logics in [BF85] give rise to AEC's?

A few examples appear in [Gro02, BL00, Bal], but there should be many more. Zilber's work on excellent classes raises several issues here [Zil05, Zil04]. He phrases his work for certain models (those satisfying the countable closure condition) in a class defined in $L_{\omega_{1}, \omega}$. So the class could be described in $L_{\omega_{1}, \omega}(Q)$; but such a formulation is of no value for the proof. The hardest part of the argument, the verification of excellence, is in the standard vein of algebraic model theory. But here infinitary conditions are being interwoven with not only algebraic but analytic arguments. Zilber's model theoretic perspective produces an intriguing group of conjectures about the complex numbers. In particular, even a very simple case of showing the complex exponential field is 'strongly exponentially closed' in the sense of [Zil04], has only been answered using Schanuel's conjecture and Hadamard factorization [Mar].

In another direction, one might try to weaken the categoricity assumption for proving tameness. The following version doesn't shed much light since we don't have any clear way at hand to verify it (aside from categoricity). Shelah called this notion rigid. I discuss this notion because a number of central steps in the analysis of categoricity in [She99], existence of non-splitting extensions, $\left(H_{1}, \lambda\right)$ tameness, and unions of saturated models are saturated are fundamentally about AEC which are epi.

Definition 14 The AEC $\boldsymbol{K}$ is epi if there is an EM-template $\Phi$ such that the functor $E M\left(_{-}, \Phi\right)$ is an epimorphism from linear orders onto the models of $\boldsymbol{K}$. 
For example, the core of the proof of Theorem 7 shows:

Corollary 15 If $\boldsymbol{K}$ is epi then $\boldsymbol{K}$ is $\left(H_{1}, \infty\right)$ - tame.

Categoricity is used in the proof of Theorem 7 to get that the AEC is epi. (Of course this terminology isn't used.)

Grossberg and VanDieren [GV] strengthen the hypothesis of Theorem 4 by adding $(\mu, \infty)$-tameness for some $\mu<\lambda$ with powerful results.

Theorem 16 [GV06a] Suppose $\boldsymbol{K}$ has the amalgamation property and arbitrarily large models. Suppose $\boldsymbol{K}$ is $\lambda$ and $\lambda^{+}$categorical for some $\lambda>\operatorname{LS}(\boldsymbol{K})$ and is $(\mu, \infty)$-tame for some $\mu<\lambda$. Then $\boldsymbol{K}$ is categorical in all cardinals above $\lambda$.

There are a number of variations on this result and on the elimination of the (categorical in $\lambda$ )-hypothesis [Les05, GV, BL00, GVV, Vana] to get 'upwards categoricity from a single cardinal' . We don't go into this further here except to remark that any full proof from these hypotheses involves an intensive investigation of EM models to show that a union of a short chain of saturated models is saturated [She99, Bal]. Natural extensions, which remain open as far as I know, are to replace categoricity in a single successor cardinal by categoricity in a regular or an arbitrary cardinal; a different idea is needed to replace the role of two cardinal models.

The stability spectrum theorem is fundamental for the study of first order theories; it is the essence of the classification of theories. But no similar result is known for general abstract elementary classes. The stability spectrum of an AEC $\boldsymbol{K}$ is the function from cardinals to cardinals which gives the supremum of the cardinals of the number of Galois types over a model in $\boldsymbol{K}$ of fixed cardinality.

Question 17 Is the stability spectra of an abstract elementary classes (even in a countable language with $\operatorname{LS}(\boldsymbol{K})=\omega$ ) one of a finite set of functions? Does $\omega$-stable imply stable in all cardinals?

Baldwin, Kueker, and VanDieren [BKV00] give a positive response to the last question but only under the extremely strong hypotheses of both $\left(\aleph_{0}, \infty\right)$ tameness and $\left(\aleph_{0}, \infty\right)$-locality. Grossberg and VanDieren earlier noted in [GV06a] (they state stronger hypotheses):

Theorem 18 Let $\mu \geq \operatorname{LS}(\boldsymbol{K})$. If $\boldsymbol{K}$ is $(\mu, \infty)$-tame and $\mu$-stable then $\boldsymbol{K}$ is stable in all $\kappa$ with $\kappa^{\mu}=\kappa$.

This follows by the standard argument after you have shown every Galois type does not $\mu$-split over a set of size $\mu$ and (using tameness) that Galois types (over appropriate base models) have unique non-splitting extensions. In the first order case, the converse to Theorem 18 requires the definition of $\kappa(T)$ and a simple argument [Bal88] uses a considerable amount of the forking calculus. A natural question is 
Question 19 What is the 'correct' notion of superstablity for AEC?

There are a number of suggestions [She99, GV, Gro02] revolving around variants in defining a concept analogous to the $\kappa(T)$ in first order logic: the length of a string of 'forking' extensions. Further complications arise from finding an appropriate notion of dependence. Below we discuss another candidate for superstability from [She01, She0x] where there is no assumption that $\boldsymbol{K}$ has arbitrarily large models. We ask naively

Question 20 Is there a $\mu$ so that stability for all $\lambda>\mu$ is a robust concept for $A E C$ ? How does it interact with the purely structural notion: Every union of a chain of saturated members of $\boldsymbol{K}$ is saturated.

While it is straightforward in regular cardinals $\kappa$ to show stability in $\kappa$ implies the existence of a saturated model in $\kappa$, considerations like those above would be necessary to extend this to any $\kappa$ in which $\boldsymbol{K}$ is stable. Note that the converse (non-stable in $\lambda$ implies no saturated model in $\lambda$ ) is a rather technical argument in the first order case.

The positive results on the categoricity spectrum for classes with arbitrarily large models depend at least indirectly on subtle applications of EM-models [Bal05]. The work of [GV06a, GV06b, GV, GVV, HV] proceeds in quite a different direction. Largely eschewing the use of EM-models the authors try to identify construction and 'stability theoretic conditions' that allow one to carry out more refined versions of the first order analysis (aiming towards geometric stability) in suitably restricted AEC.

Grossberg and VanDieren originated this trend in their analysis of the stability spectrum for tame AEC in [GV06a]; it continued in further work on the stability spectrum [BKV00] and the analysis of categoricity in [GV06b, GV, BL00] and under even stronger hypotheses in [HV, Hyt]. This kind of work suggests several directions of inquiry.

Some of the crucial notions in this development are limit models, a new notion of strong type, towers of models and the means for analyzing them, variations on splitting (with assorted cardinal parameters). Determining the interrelations of these notions provides a fertile field of study. The notion of limit model is essential for studying structures with cardinality $\operatorname{LS}(\boldsymbol{K})$ because under the usual notion of Galois type (the domain of a type must be a model), the concept of a saturated model in cardinality $\operatorname{LS}(\boldsymbol{K})$ is vacuous.

The work of $[\mathrm{HV}]$ is novel as it introduces a notion of type defined by mappings (i.e. a kind of Galois type) but considers types over arbitrary subsets. Key to this is the observation that the proof of transitivity in establishing an equivalence relation by $\operatorname{tp}_{A}(a)=\operatorname{tp}_{B}(b)$ if there is an automorphism of the universe taking $a$ to $b$ uses amalgamation not of the domain models (in the sense of Shelah's definition [She83a, She99, Gro02]) but rather amalgamation of the models in which the types are realized. They work in the general context of AEC with arbitrarily large models and amalgamation and make the additional assumptions that $\boldsymbol{K}$ has a prime model and extend amalgamation 
to disjoint amalgamation. Making effective use of this notion of Galois type requires the condition we'll call $H$-local: if $A \subset B$ and for every finite $\boldsymbol{a}$ in $A, \operatorname{tp}_{A}(\boldsymbol{a})=\operatorname{tp}_{B}(\boldsymbol{a})$, then $A \prec \boldsymbol{K} B$. This condition is easily seen to hold for all syntactically defined AEC (e.g. models of an arbitrary sentence of $L_{\omega_{1}, \omega}$.) They further prove that for tame AEC satisfying their general conditions, their notion of weak type coincides with Galois types. (In general $\boldsymbol{a}$ and $\mathbf{b}$ have the same Galois type implies they have same weak type implies they have the same syntactic type.)

One can ask for mathematical examples to justify the study of particular families of hypotheses. For example,

Question 21 Is there an example of an $A E C \boldsymbol{K}$ with $\operatorname{LS}(\boldsymbol{K})=\omega$ which is $\left(\aleph_{0}, \infty\right)$-tame and is not defined by a sentence in $L_{\omega_{1}, \omega}(Q)$ ?

Excellence is another condition to impose; in [GH89], the class $\boldsymbol{K}$ is defined in $L_{\omega_{1}, \omega}$. Excellence requires a notion of independence; essentially excellence consists in requiring the existence of 'prime models' over independent $n$-cubes. See [Bal04] for an intuitive introduction. Grossberg and Hart [GH89] prove a 'main gap' theorem in their context. It would be interesting to try to replace $\omega$ stable (part of the definition of excellence in this context) by stable. Grossberg, Kolesnikov and Lessmann [GK, Kol05, GL05] deal with AEC that are equipped with an independence notion. Thus, this work is in the tradition of [She87, BS90] and has some similarity to Shelah's [She0x, She00b] study of frames.

Much of the work described here has been under the hypothesis of amalgamation. Naturally, the original more general question remains.

Question 22 Explore AEC which have arbitrarily large models but without assuming the amalgamation property.

Shelah and Villaveces [SV99] and VanDieren [Vanb] weaken 'amalgamation property' to 'no maximal models'. With the use of the Devlin-Shelah diamond they are able to prove the existence of a 'dense' family of amalgamation bases and carry over much of the analysis. In [Vanb], under further model theoretic hypotheses, the uniqueness of limit models is established. With the increased understanding of categoricity transfer problem for classes with amalgamation, the following is much more accessible.

Question 23 Prove Theorem 4 and Theorem 16, but weakening the hypothesis of amalgamation property to no maximal models. To get the full information we have for classes with amalgamation, prove (an appropriate variant) of Theorem 7 under the weaker hypothesis.

The study of infinitary logic often appeared to have a heavy dependence on axiomatic set theory. This was perhaps exacerbated by studies (e.g. [MS90, KS96]) of logics $L_{\kappa, \omega}$ or $L_{\kappa, \kappa}$ where $\kappa$ was a large (compact or measurable) cardinal. But some extensions beyond $\mathrm{ZFC}$ are needed for the harder theme in studying AEC; we no longer assume that $\boldsymbol{K}$ has arbitrarily large models. Certain landmark results depend on set theory. In particular, Shelah originally assumed $\diamond$ to prove: 
Theorem 24 [She75] If a sentence of $L_{\omega_{1}, \omega}(Q)$ is $\aleph_{1}$-categorical then it has a model of power $\aleph_{2}$.

But, in [She87] and as expounded in [Gro02, Bal] the result can be given an extremely beautiful proof in ZFC; it is not quite possible to describe the result just in the language of AEC's [She, Bal]. In contrast two other results:

Theorem 25 Suppose $2^{\aleph_{0}}<2^{\aleph_{1}}$.

1. If the $A E C \boldsymbol{K}$ is $\aleph_{1}$-categorical then $\boldsymbol{K}$ is $\omega$-stable.

2. Suppose $2^{\lambda}<2^{\lambda^{+}}$. If the AEC $\boldsymbol{K}$ is $\lambda$-categorical, with $\lambda \geq \operatorname{LS}(\boldsymbol{K})$ but fails the amalgamation property in $\lambda$ then it has $2^{\lambda^{+}}$models of cardinality $\lambda^{+}$.

actually require the set theoretic hypothesis. This necessity is outlined in [She87], and more clearly in the revised version of that paper [She]; see [Gro02] for a good account of the positive Theorem 252 ). Complete proofs of both results including finding counterexamples in $L_{\omega_{1}, \omega}$ showing the necessity of weak $\mathrm{CH}$ appear in [Bal]. The most striking result which has no apparent upward Löwenheim-Skolem assumption is Shelah's proof of the appropriate version of the Morley conjecture for $L_{\omega_{1}, \omega}$ :

Theorem 26 [She83a, She83b] Assume for each $n<\omega, 2^{\aleph_{n}}<2^{\aleph_{n+1}}$. If $\psi$ is a sentence in $L_{\omega_{1}, \omega}$ in a countable vocabulary that is categorical in all cardinals less than $\aleph_{\omega}$ the $\psi$ defines an excellent class that is categorical in all cardinals.

The only full account of this is [She83a, She83b]; there are several accounts of the deduction of categoricity from excellence (e.g. [Les, Bal]. The excellence is actually derived from the hypothesis that there are less than the maximum number of models in each cardinal below $\aleph_{n}$ (there is a further set theoretic hypothesis hidden here; 'maximum' is a little more complicated than usual; see [She83a, She83b]).

The $L_{\omega_{1}, \omega}$ work depends heavily the assumption that $\operatorname{LS}(\boldsymbol{K})=\omega$ and for this reason it does not apply nor extend in a straightforward manner to $L_{\omega_{1}, \omega}(Q)$. More generally, to extend this kind of result to $\boldsymbol{K}$ with $\operatorname{LS}(\boldsymbol{K})>\omega$ requires different methods. Shelah has a number of works in this area, which have not yet been published. Assuming, $2^{\lambda}<2^{\lambda^{+}}<2^{\lambda^{+}}$, Shelah asserts in the introduction to [She01] that categoricity of an AEC in three successive cardinals implies the existence of a model in the next cardinal. Further, he asserts that categoricity in the third cardinal can be replaced by 'fewer than the maximal number of model. Further works approaches the goal of showing that for an arbitrary AEC, categoricity on a sufficiently long interval of cardinals implies the existence of arbitrarily large models.

Shelah's work [She0x, She00a, She00b] introduces the notion of a frame and the stronger notion of a good frame. He regards this as a notion of 'superstability' for this context. In short, a frame describes more extensive conditions 
on the models in an AEC $\boldsymbol{K}$ of cardinality $\kappa$ which suffice to move upward (say from categoricity in $\kappa$ to existence or uniqueness of models in larger powers). In the other direction, the existence of a frame in cardinality $\kappa$ is derived from categoricity in cardinals above $\kappa$; a major theme is to reduce the number of cardinals above $\kappa$ in which one must make the categoricity hypothesis.

In one direction one can hope to generalize to AEC the fundamental results of first order stability theory; to classify AEC by some kind of stability notion, develop a robust notion of independence, and compute the possible spectra of an AEC under natural conditions.

A greater challenge is to relate this general study more directly to problems from mainstream mathematics. Broadly speaking, in the first order case, it was found that the studying the first order theory of a structure, the reals, the complexes, and others allowed one to get serious information about the structure. If the most optimistic scenario concerning Zilber's conjectures worked out, there would be evidence for the $L_{\omega_{1}, \omega}(Q)$-theory playing a similar role. But there is no candidate at present for associating a more general AEC with a structure. It seems more likely to me that the significance of these ideas will rest more in their role of enabling us to understand that Cantor's paradise is not merely combinatorial; rather future generations will understand a rich world of complex mathematical structures of unlimited cardinality. In particular, the fact that combinatorial principles (Devlin-Shelah diamond) which are derived from the weak GCH, allow the development of a smooth model theory may eventually be viewed as evidence for the naturality of the weak GCH. Further, as we begin to consider in depth structures with cardinality beyond the continuum, we may focus even more on considering naturally defined classes of structures rather than individual structures.

\section{References}

[Bal] John T. Baldwin. Categoricity. www.math.uic.edu ${ }^{\sim}$ jbaldwin.

[Bal88] J.T. Baldwin. Fundamentals of Stability Theory. Springer-Verlag, 1988.

[Bal04] J.T. Baldwin. Notes on quasiminimality and excellence. Bulletin of Symbolic Logic, 10:334-367, 2004.

[Bal05] J.T. Baldwin. Ehrenfeucht-Mostowski models in abstract elementary classes. In Yi Zhang, editor, Logic and its Applications, Contemporary Mathematics, pages 1-17. American Mathematical Society, 2005.

[BF85] J. Barwise and S. Feferman, editors. Model-Theoretic Logics. SpringerVerlag, 1985.

[BH00] J.T. Baldwin and K. Holland. Constructing $\omega$-stable structures: Rank 2 fields. The Journal of Symbolic Logic, 65:371-391, 2000. 
[BKV00] J.T. Baldwin, D.W. Kueker, and M. VanDieren. Upward stability transfer theorem for tame abstract elementary classes. to appear: Notre Dame Journal of Formal Logic, 200?

[BL00] J.T. Baldwin and O. Lessmann. Upward categoricity of very tame abstract elementary classes with amalgamation. to appear; APAL, $200 ?$

[BL03] S. Buechler and O. Lessmann. Simple homogeneous models. Journal of the American Mathematical Society, 16:91-121, 2003.

[BS] J.T. Baldwin and S. Shelah. Examples of non-locality. submitted.

[BS90] J.T. Baldwin and S. Shelah. The primal framework: I. Annals of Pure and Applied Logic, 46:235-264, 1990.

[BY03a] I. Ben-Yaacov. Positive model theory and compact abstract theories. Journal of Mathematical Logic, 3:85-118, 2003.

[BY03b] I. Ben-Yaacov. Simplicity in compact abstract theories. Journal of, 3:163-191, 2003.

[EM90] P. Eklof and Alan Mekler. Almost Free Modules: Set theoretic Methods. North Holland, 1990.

[GH89] Rami Grossberg and Bradd Hart. The classification theory of excellent classes. The Journal of Symbolic Logic, 54:1359-1381, 1989.

[GK] Rami Grossberg and Alexei Kolesnikov. Excellent abstract elementary classes are tame. preprint.

[GL05] Rami Grossberg and Olivier Lessmann. Abstract decomposition theorem and applications. In Logic and its Applications, Contemporary Mathematics, pages 73-108. AMS, 2005.

[Gro02] Rami Grossberg. Classification theory for non-elementary classes. In Yi Zhang, editor, Logic and Algebra, pages 165-204. AMS, 2002. Contemporary Mathematics 302.

[GS83] Rami Grossberg and Saharon Shelah. On universal locally finite groups. Israel J Math, 44:289-302, 1983).

[GV] R. Grossberg and M. VanDieren. Categoricity from one successor cardinal in tame abstract elementary classes. preprint.

[GV06a] R. Grossberg and M. VanDieren. Galois stability for tame abstract elementary classes. Journal of Mathematical Logic, 6:1-24, 2006.

[GV06b] R. Grossberg and M. VanDieren. Shelah's categoricity conjecture from a successor for tame abstract elementary classes. The Journal of Symbolic Logic, 71:553-568, 2006. 
[GVV] R. Grossberg, M. VanDieren, and Andrés Villaveces. Limit models in stable classes with amalgamation. preprint.

[Hen72] W. Henson. Countable homogeneous relational structures and $\aleph_{0^{-}}$ categorical theories. The Journal of Symbolic Logic, 37:494-500, 1972.

[HI02] W. Henson and Jose Iovino. Ultraproducts in analysis. In Analysis and Logic, volume 262 of London Mathematical Society Lecture Notes, pages 1-115. Cambridge University Press, 2002.

[Hru92] E. Hrushovski. Strongly minimal expansions of algebraically closed fields. Israel Journal of Mathematics, 79:129-151, 1992.

[Hru93] E. Hrushovski. A new strongly minimal set. Annals of Pure and Applied Logic, 62:147-166, 1993.

[HS90] Bradd Hart and Saharon Shelah. Categoricity over $P$ for first order $T$ or categoricity for $\phi \in 1_{\omega_{1} \omega}$ can stop at $\aleph_{k}$ while holding for $\aleph_{0}, \cdots, \aleph_{k-1}$. Israel Journal of Mathematics, 70:219-235, 1990.

[HV] T. Hyttinen and M. Viljanen. Independence in local aec: Part i. preprint.

[Hyt] T. Hyttinen. Uncountably categorical local tame abstract elementary classes with disjoint amalgamation. preprint.

[Kol05] Alexei Kolesnikov. Dependence relations in non-elementary classes. In Logic and its Applications, Contemporary Mathematics, pages 203231. AMS, 2005.

[KP97] Byunghan Kim and Anand Pillay. Simple theories. Annals of Pure and Applied Logic, 88:149-164, 1997.

[KS96] Oren Kolman and Saharon Shelah. Categoricity of theories in $l_{\kappa \omega}$, when $\kappa$ is a measureable cardinal, part I. Fundamenta Mathematica, 151:209-240, 1996.

[Les] Olivier Lessmann. Categoricity and U-rank in excellent classes. to appear, Journal of Symbolic Logic.

[Les05] Olivier Lessmann. Upward categoricity from a successor cardinal for an abstract elementary class with amalgamation. Journal of Symbolic Logic, 70:639-661, 2005.

[LP] C. Laskowski and A. Pillay. Morley's theorem for gross models. preprint.

[Mar] D. Marker. A remark on Zilber's pseudo-exponentiation. preprint.

[Moo] R. Moosa. On saturation and the model theory of compact Kahler manifolds. to appear. 
[MS90] Michael Makkai and Saharon Shelah. Categoricity of theories in $l_{\kappa \omega}$, with $\kappa$ a compact cardinal. Annals of Pure and Applied Logic, 47:4197, 1990 .

[PW0x] M. Pourmahdian and F. Wagner. A simple positive Robinson theory with Lstp $\neq$ Stp. In Mojtaba Moniri and, editor, Model theory Confernce in Tehran. 200x.

[Rad04] Dale Radin. A definability result for compact complex spaces. $J$. Symbolic Logic, 69:241-254, 2004.

[She] Saharon Shelah. Abstract elementary classes near $\aleph_{1}$ sh88r. revision of Classification of nonelementary classes II, Abstract elementary classes; consult Shelah for copy.

[She70] S. Shelah. Finite diagrams stable in power. Annals of Mathematical Logic, 2:69-118, 1970.

[She75] S. Shelah. Categoricity in $\aleph_{1}$ of sentences in $L_{\omega_{1}, \omega}(Q)$. Israel Journal of Mathematics, 20:127-148, 1975. paper 48.

[She80] Saharon Shelah. Simple unstable theories. Annals of Mathematical Logic, 19:177-203, 1980.

[She83a] S. Shelah. Classification theory for nonelementary classes. I. the number of uncountable models of $\psi \in L_{\omega_{1} \omega}$ part A. Israel Journal of Mathematics, 46:3:212-240, 1983. paper 87a.

[She83b] S. Shelah. Classification theory for nonelementary classes. I. the number of uncountable models of $\psi \in L_{\omega_{1} \omega}$ part B. Israel Journal of Mathematics, 46;3:241-271, 1983. paper 87b.

[She87] Saharon Shelah. Classification of nonelementary classes II, abstract elementary classes. In J.T. Baldwin, editor, Classification theory (Chicago, IL, 1985), pages 419-497. Springer, Berlin, 1987. paper 88: Proceedings of the USA-Israel Conference on Classification Theory, Chicago, December 1985; volume 1292 of Lecture Notes in Mathemat$i c s$.

[She99] S. Shelah. Categoricity for abstract classes with amalgamation. Annals of Pure and Applied Logic, 98:261-294, 1999. paper 394. Consult Shelah for post-publication revisions.

[She00a] S. Shelah. On categorical a.e.c. paper 734 in preparation, 200?

[She00b] S. Shelah. Toward classification theory of good $\lambda$ frames and abstract elementary classes. preprint 705, 200?

[She01] S. Shelah. Categoricity of abstract elementary class in two successive cardinals. Israel Journal of Mathematics, 126:29-128, 2001. paper 576 . 
[She0x] S. Shelah. Categoricity of abstract elementary classes: going up inductive step. preprint $600,200 x$.

[SV99] Saharon Shelah and Andrés Villaveces. Toward categoricity for classes with no maximal models. Annals of Pure and Applied Logic, 97:1-25, 1999. revised version: www.math.rutgers. edu/ ${ }^{\text {shelah. }}$

[Vana] Monica VanDieren. Categoricity and no Vaughtian pairs. preprint.

[Vanb] Monica VanDieren. Categoricity in abstract elementary classes with no maximal models. to appear.

[Zil00] B.I. Zilber. Covers of the multiplicative group of an algebraically closed field of characteristic 0. to appear JLMS, 2000.

[Zil02] B.I. Zilber. Raising to powers in algebraically closed fields. preprint, 2002.

[Zil04] B.I. Zilber. Pseudo-exponentiation on algebraically closed fields of characteristic 0. Annals of Pure and Applied Logic, 132:67-95, 2004.

[Zil05] B.I. Zilber. A categoricity theorem for quasiminimal excellent classes. In Logic and its Applications, Contemporary Mathematics, pages 297306. AMS, 2005. 